\title{
Nrf2 induces Ucp1 expression in adipocytes in response to $\beta 3$-AR stimulation and enhances oxygen consumption in high-fat diet-fed obese mice
}

\author{
Seo-Hyuk Chang ${ }^{1}$, Jaeyool Jang ${ }^{1}$, Seungjun Oh ${ }^{1}$, Jung-Hoon Yoon ${ }^{1}$, Dong-Gyu Jo ${ }^{2}$, Ui Jeong Yun ${ }^{1, *} \mathcal{E}^{*}$ Kye Won Park ${ }^{1, *}$ \\ ${ }^{1}$ Department of Food Science and Biotechnology and ${ }^{2}$ School of Pharmacy, Sungkyunkwan University, Suwon 16419, Korea
}

\begin{abstract}
Cold-induced norepinephrine activates $\beta 3$-adrenergic receptors (B3-AR) to stimulate the kinase cascade and cAMP-response element-binding protein, leading to the induction of thermogenic gene expression including uncoupling protein 1 (Ucp1). Here, we showed that stimulation of the $\beta 3-\mathrm{AR}$ by its agonists isoproterenol and CL316,243 in adipocytes increased the expression of Ucp1 and Heme Oxygenase 1 (Hmox1), the principal Nrf2 target gene, suggesting the functional interaction of $\mathrm{Nrf} 2$ with $\beta 3-\mathrm{AR}$ signaling. The activation of $\mathrm{Nrf} 2$ by tert-butylhydroquinone and reactive oxygen species (ROS) production by glucose oxidase induced both Ucp1 and Hmox1 expression. The increased expression of Ucp1 and Hmox1 was significantly reduced in the presence of a Nrf2 chemical inhibitor or in Nrf2-deleted (knockout) adipocytes. Furthermore, Nrf2 directly activated the Ucp1 promoter, and this required DNA regions located at -3.7 and $-2.0 \mathrm{~kb}$ of the transcription start site. The CL316,243induced Ucp1 expression in adipocytes and oxygen consumption in obese mice were partly compromised in the absence of Nrf2 expression. These data provide additional insight into the role of Nrf2 in $\beta 3$-AR-mediated Ucp1 expression and energy expenditure, further highlighting the utility of Nrf2-mediated thermogenic stimulation as a therapeutic approach to diet-induced obesity. [BMB Reports 2021; 54(8): 419-424]
\end{abstract}

\section{INTRODUCTION}

The uncoupling of respiration and ATP synthesis contribute to non-shivering thermogenesis in adipose tissues during chronic cold exposure $(1,2)$. The respiratory chain in mitochondria

*Corresponding authors. Ui Jeong Yun, Tel: +82-31-290-7908; Fax: +82-31-290-5270; E-mail: yunc@skku.edu; Kye Won Park, Tel: +8231-290-7804; Fax: +82-31-290-7882; E-mail: kwpark@skku.edu

https://doi.org/10.5483/BMBRep.2021.54.8.023

Received 16 February 2021, Revised 8 March 2021, Accepted 9 March 2021

Keywords: $\beta 3$-adrenergic receptor, Energy expenditure, Nrf2, Obesity, Uncoupling protein 1 accumulates protons $(\mathrm{H}+)$ in the intermembrane space that fall into ATP synthase to generate ATP. During cold exposure, the accumulated protons in the intermembrane space are dissipated by uncoupling protein 1 (Ucp1) and produce heat instead of ATP $(3,4)$. Thermogenic adipocytes expressing Ucp1 mainly found in brown adipose tissue (BAT) and subcutaneous white adipose tissue (WAT) is stimulated by $\beta$-adrenergic receptor ( $\beta 3$-AR) activation $(2,5-8)$.

Cold stress induces the secretion of norepinephrine, leading to the activation of $\beta 3$-ARs in adipose tissue $(9,10)$. $\beta 3$-AR regulates whole-body thermogenesis in part through Ucp1 stimulation in adipose tissue (11). BAT in Ucp1 knockout (KO) mice has been reported to be unresponsive to norepinephrine $(12,13)$, suggesting the importance of Ucp1 in $\beta 3$-AR signaling-mediated thermogenesis. Stimulation of $\beta 3$-ARs by agonists induces the $\mathrm{G}_{\mathrm{s}}$-dependent activation of adenylate cyclase, increases intracellular cAMP levels, and activates protein kinase A (PKA) (10, 14). Activated PKA increases the transcription of various target genes through transcription factor cAMP-responsive element-binding protein (Creb). Creb binds to the promoter region of target genes containing the cAMP-responsive element (CRE) (15).

Nuclear factor erythroid 2-related factor 2 (Nrf2), a master regulator of the anti-oxidant response, removes excess reactive oxygen species (ROS) by inducing antioxidant-related genes under oxidative stress conditions $(16,17)$. Nrf2 binds to the antioxidant response element (ARE) of the target genes to induce the transcription of cytoprotective antioxidation-related genes such as Heme Oxygenase-1 (Hmox1), NAD(P)H quinone oxidoreductase 1 (Nqo1), and glutamate-cysteine ligase modifier subunit (Gclm), and glutathione reductase (16). Previous studies showed that Nrf2 interact with other signaling pathways including the arylhydrocarbon receptor, NF- $\mathrm{KB}, \mathrm{NFATC1}$, and p53 $(18,19)$. Thus, it is possible that Nrf2 acts cooperatively with intracellular signaling pathways responding to various cellular stresses.

In this study, we examined the potential roles of Nrf2 in $\beta 3$-AR-mediated Ucp1 expression in adipocytes. We showed that the $\beta 3$-AR-induced $U_{c p} 1$ expression and stimulated oxygen consumption was partly impaired in Nrf2 KO obese mice. These studies indicate the novel role of Nrf2 in $\beta 3-\mathrm{AR}$ activation in adipocytes.

ISSN: 1976-670X (electronic edition)

Copyright (C) 2021 by the The Korean Society for Biochemistry and Molecular Biology

(c) This is an open-access article distributed under the terms of the Creative Commons Attribution Non-Commercial License (http://creativecommons.org/licenses/by-nc/4.0) which permits unrestricted non-commercial use, distribution, and reproduction in any medium, provided the original work is properly cited. 


\section{RESULTS}

\section{B3-AR activation induces both Ucp1 and Hmox1 expression in adipocytes}

To examine the possible interactions between $\mathrm{Nrf} 2$ and $\beta 3-\mathrm{AR}$ signaling in adipocytes, we stimulated adipocytes with $\beta 3$-AR agonists and measured the expression of Hmox1, the principal Nrf2 target gene in adipocytes. Strong Ucp1 mRNA induction was observed within $1 \mathrm{~h}$ and reached maximal levels of $\sim 50$-fold at $3 \mathrm{~h}$. Similarly, time-dependent increases in Hmox 1 mRNA levels were observed in $\mathrm{C} 3 \mathrm{H} 10 \mathrm{~T} 1 / 2$ adipocytes (Fig. 1A). Treatment of primary adipocytes isolated from inguinal white adipose tissue with CL316,243 also induced Ucp1 and Hmox 1 expression (Fig. 1B). We also confirmed that the treatment of $\mathrm{C} 3 \mathrm{H} 10 \mathrm{~T} 1 / 2$ adipocytes with another $\beta$-AR agonist isoproterenol stimulated Hmox1 and Ucp1 expression (Fig 1C). Adipocytes exposed to cool temperatures at $30^{\circ} \mathrm{C}$ similarly induced both Ucp1 and Hmox1 expression (Fig. 1D). These data suggest an interaction between Nrf2 and $\beta 3-A R$ signaling in adipocytes.

\section{Nrf2 activation mimics $\beta 3-A R$ stimulated-Ucp1 expression in adipocytes}

Given the Ucp 1 and Hmox 1 induction by $\beta 3-A R$ activation in adipocytes, we investigated whether Nrf2 activation would promote Ucp1 expression similar to that of $\beta 3$-AR activators. We found that pharmacological Nrf2 activation by tert-butylhydroquinone (tBHQ) also stimulated Hmox 1 and $U_{c p} 1$ expression (Fig. 2A, B).

An increase in oxidative stress due to the generation of ROS leads to the activation of Nrf2 (20). To determine whether ROS-

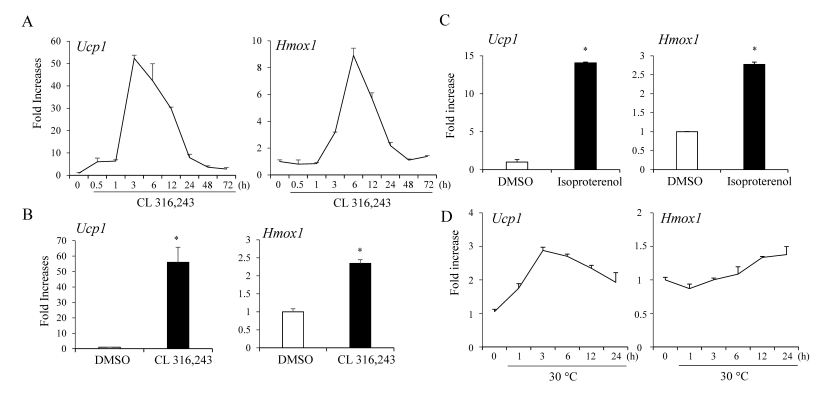

Fig. 1. $\beta 3-A R$ activation induces $U c p 1$ and $H \operatorname{mox} 1$ expression in adipocytes. (A) Time-dependent increases in Ucp1 and Hmox1 mRNA levels by treatment with CL316,243 (5 $\mu \mathrm{M})$ expressed as fold-increases relative to levels in dimethyl sulfoxide (DMSO)-treated C3H10T1/2 adipocytes. (B) Treatment of primary adipocytes isolated from inguinal adipose tissues with CL316,243 (5 $\mu \mathrm{M})$ for $6 \mathrm{~h}$. (C) Treatment of $\mathrm{C} 3 \mathrm{H} 10 \mathrm{~T} 1 / 2$ adipocytes with isoproterenol at $10 \mathrm{nM}$ for $6 \mathrm{~h}$. (D) Differentiated C3H10T1/2 adipocytes were exposed to $37^{\circ} \mathrm{C}$ or $30^{\circ} \mathrm{C}$ and the expression of $U_{c p} 1$ and Hmox 1 was measured. The data represent means \pm s.e.m. and are representative of three independent experiments. Statistical significance was determined relative to controls using the Student's $t$-test ( $\left.{ }^{*} P<0.05\right)$. induced Nrf2 activation affected Ucp1 and Hmox1 expression, we treated cells with glucose oxidase to generate ROS (21) and measured Hmox1 and Ucp1 expression. Consistently, treatment with glucose oxidase increased Ucp1 and Hmox1 levels similar to those from $\mathrm{tBHQ}$ treatment (Fig. 2C, D).

Because Nrf2 activation induced Ucp1 expression, we further investigated the effect of Nrf2 on human Ucp1promoter activity. Toward this, we cloned the proximal $3.7 \mathrm{~kb}$ of the human Ucp1 promoter into the PGL-3 vector to obtain Ucp1 promoter-driven luciferase reporter constructs. Transient Nrf2 expression stimulated $-3.7 \mathrm{~kb}$ Ucp1 promoter driven luciferase activity, whereas Nrf2 did not promote luciferase activity in the $-2.0 \mathrm{~kb}$ and $-1.0 \mathrm{~kb}$ Ucp1 proximal promoter (Fig. 2E). These data indicate that Nrf2 increases Ucp1 transcription and that this stimulatory effect required proximal promoter regions located at -3.7 and $-2.0 \mathrm{~kb}$ of the transcription start site.

\section{Nrf2 is essential for the tBHQ (Nrf2 activator) induced Ucp1 expression in adipocytes}

The effects of Nrf2 activation in adipocytes suggested that Nrf2 was essential for the effects of $\mathrm{tBHQ}$ on adipocytes. To test this, we treated the cells with $\mathrm{N}$-acetylcysteine (NAC), a known

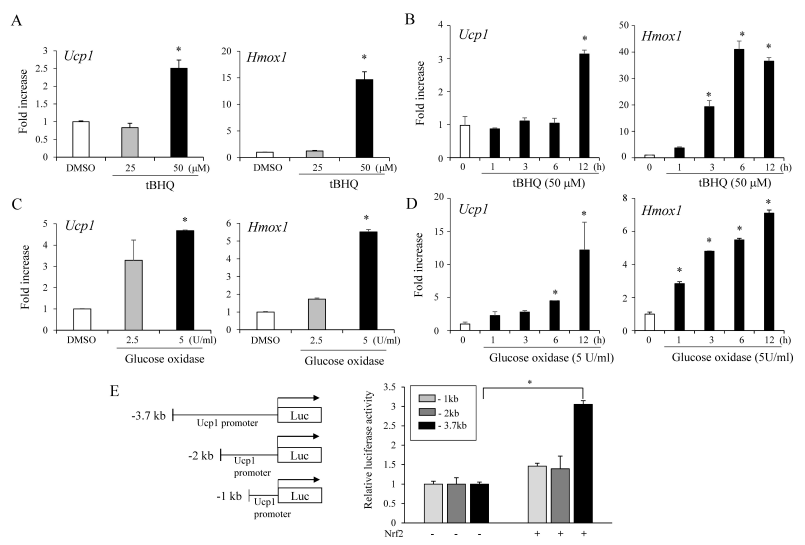

Fig. 2. Nrf2 activation increases Ucp1 expression in adipocytes. (A) tert-Butylhydroquinone ( $\mathrm{tBHO}$ ) at 25 and $50 \mu \mathrm{M}$ increases Ucp 1 and Hmox1 expression in C3H10T1/2 adipocytes. (B) Timedependent increases in Ucp 1 and Hmox 1 mRNA levels by treatment with $50 \mu \mathrm{M} \mathrm{tBHQ}$ expressed as fold-increases relative to the levels in DMSO-treated C3H10T1/2 adipocytes. (C) Treatment with glucose oxidase $(2.5 \mathrm{U}$ or $5 \mathrm{U} / \mathrm{ml})$ increases Ucp 1 and Hmox 1 expression in C3H10T1/2 adipocytes. (D) Time-dependent increases in Ucp1 and Hmox 1 mRNA levels by $5 \mathrm{U} / \mathrm{ml}$ glucose oxidase expressed as fold-increases relative to levels in DMSO-treated C3H10T1/2 adipocytes. (E) Nrf2 activates the Ucp1 promoter in the reporter assay. 293FT cells were transiently transfected with Ucp1 promoter-luciferase with or without the Nrf2-expressing vector. The proximal $3.7 \mathrm{~kb}$, $2 \mathrm{~kb}$, or $1 \mathrm{~kb}$ of the human Ucp1 promoter sequences were fused to express the Ucp1 promoter-driven luciferase reporter. The data represent averages $+/-$ s.e.m. of triplicates and are expressed as foldincreases relative to empty vector-transfected cells. Statistical significance was determined relative to controls using the Student's $t$-test $(* P<0.05)$. 
ROS-scavenging chemical, to inhibit Nrf2 activity, and assessed its effects. Treatment with NAC has been shown to decrease Nrf2 activity (22). Consistently, NAC impaired the tBHQinduced expression of $U_{c p} 1$ and Nrf2 target genes (Fig. 3A).

To further show the role of Nrf2 in Ucp1 induction, we treated primary adipocytes isolated from the inguinal fat pads of wild-type (Nrf2 WT) and Nrf2 knockout (Nrf2 KO) mice with $\mathrm{tBHQ}$. Nrf2 activation strongly induced the Ucp1 and Nrf2 target gene expression of Hmox1, Nqo1, Srxn1, and Gclc in Nrf2 WT primary adipocytes. However, these effects were significantly impaired in the Nrf2 KO adipocytes (Fig. 3B). Together, these data show that Nrf2 activation can induce Ucp1 expression in adipocytes.

\section{$\beta 3-A R$ induced oxygen consumption is partly dependent on Nrf2}

The ability of $\beta 3-A R$ to increase Hmox 1 and Ucp1 expression suggested that Nrf2 might be involved in $\beta 3$-AR-induced Ucp1 expression in adipocytes. To investigate this possibility, we treated Nrf2 WT and Nrf2 KO adipocytes with CL316,243. B3-AR activation strongly induced the expression of $U_{c p} 1$ and $\mathrm{Nrf} 2$ target genes in Nrf2 WT adipocytes. However, these effects were significantly blunted in Nrf2 $\mathrm{KO}$ adipocytes (Fig. 4A).

To further assess the role of $\mathrm{Nrf} 2$ in $\beta 3$-AR activation in mice, we measured the CL316,243-induced $\mathrm{O}_{2}$ consumption rates of aged WT and Nrf2 KO female mice. CL316,243 treatment of 14-month-old mice increased oxygen consumption by about $123 \%$ compared to the basal levels in WT mice,

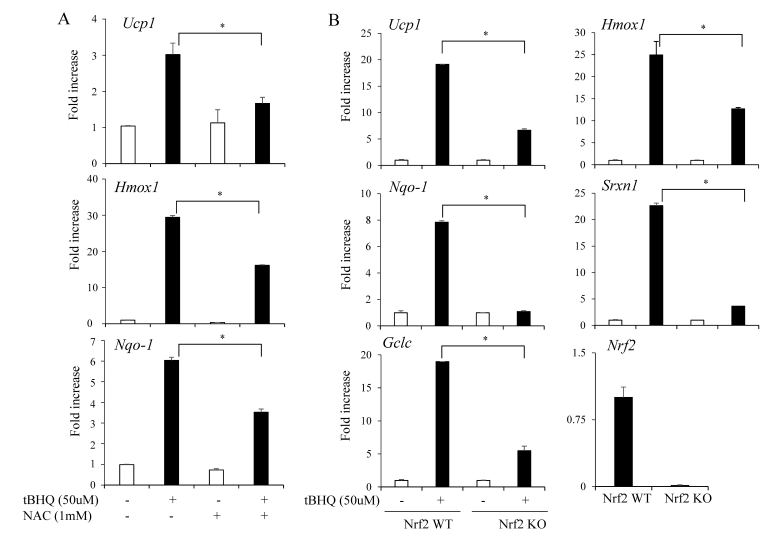

Fig. 3. Nrf2 is essential for tert-butylhydroquinone (tBHQ)-mediated Ucp1 induction in adipocytes. (A) C3H10T1/2 adipocytes were treated with tBHQ $(50 \mu \mathrm{M})$ and NAC (1 mM) for $12 \mathrm{~h}$ and the expression of Ucp1, Hmox1, and Nqo1 was measured. (B) Primary adipocytes isolated from the inguinal fat pads of Nrf2 wild-type (Nrf2 WT) and Nrf2 knockout mice (Nrf2 KO) were treated with tBHQ $(50 \mu \mathrm{M})$ for $12 \mathrm{~h}$ and the expression levels of Ucp1 and Nrf2 target genes (Hmox1, Nqo1, Srxn1, and Gclc) were measured. The data represent means \pm s.e.m. and are representative of three independent experiments. Statistical significance was determined relative to controls using the Student's t-test $(* P<0.05)$. whereas CL316,243 exhibited 110\% increases in Nrf2 KO aged female mice (Fig. 4B). We also measured $\beta 3$-AR-induced oxygen consumption in obese C57BL/6J male mice fed high-fat diet (HFD) for 16 weeks. The WT obese mice stimulated with CL316, 243 showed increased rates of oxygen consumption up to $120 \%$ but its stimulatory effects were less $(113 \%)$ in the Nrf2 $\mathrm{KO}$ obese mice (Fig. 4C). These findings suggest the role of $\mathrm{Nrf2}$ in $\beta 3$-AR-mediated oxygen consumption. Increased metabolic activity by $\beta 3-A R$ stimulation increases body temperature. Thus, we further investigated rectal temperatures for $24 \mathrm{~h}$ in the Nrf2 WT and Nrf2 KO obese mice. Consistent with the oxygen consumption, the nighttime (at 4 A.M) rectal temperature in Nrf2 WT mice was significantly higher than that in the Nrf2 KO mice (Fig. 4D). Together, these data strongly suggest that Nrf2 was partly involved in the $\beta 3-A R-U c p 1$ axis in certain conditions and further suggest that adipose Nrf2 activation can be a regulator of the energy expenditure of obese or aged mice.

\section{DISCUSSION}

In oxidative stress conditions, Nrf2 is activated to induce the expression of cytoprotective genes (16). It has become evident that Nrf2 is interconnected with other signaling pathways such as NF- $\mathrm{KB}$ and p53 (18). Furthermore, Nrf2 may also exert its effects through interactions with additional pathways such as the PKA/Creb signaling pathway. Nrf2 in fasting conditions becomes activated by the cAMP/PKA pathway and regulates its downstream target genes in hepatocytes (23). CREB signaling was also shown to increase Hmox 1 in mouse adrenal cells and

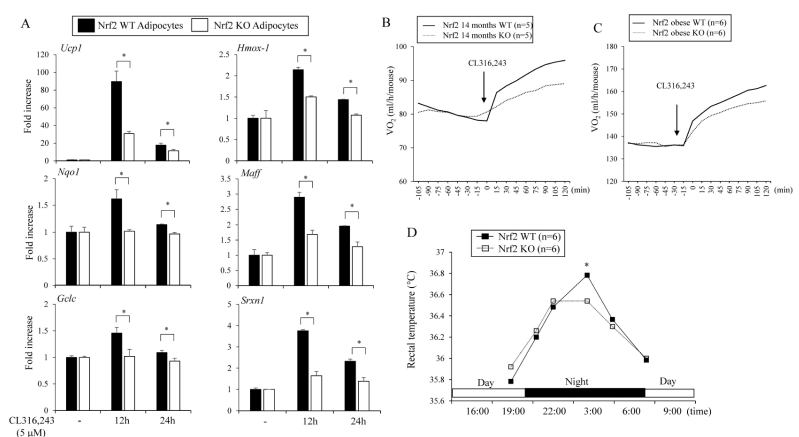

Fig. 4. $\beta 3-A R$-stimulated Ucp1 induction and oxygen consumption are partly dependent upon Nrf2. (A) Primary adipocytes isolated from the inguinal fat pads of Nrf2 WT and Nrf2 KO mice were treated with CL316,243 $(5 \mu \mathrm{M})$ for 12 or $24 \mathrm{~h}$ and the expression levels of Ucp1 and Nrf2 target genes (Hmox1, Nqo1, Maff, Gclc, and Srxn1) were measured. (B) Fourteen-month-old Nrf2 WT and $\mathrm{Nif} 2 \mathrm{KO}$ female mice were stimulated with $\beta 3$-AR agonist (CL316,243, $2 \mathrm{mg} / \mathrm{kg}$ ) and the $\mathrm{O}_{2}$ consumption rates were measured. (C) The $\mathrm{O}_{2}$ consumption rates of Nrf2 WT and Nrf2 KO obese male mice fed high-fat diet for 16 weeks were measured. (D) Rectal temperature was measured for $24 \mathrm{~h}$ in WT and Nrf2 KO obese mice. The data represent mean \pm s.e.m. and statistically significant differences between WT and Nrf2 KO mice were determined by the Student's t-test $* P<0.05)$. 
human umbilical vein endothelial cells (24-26). Therefore, these studies raise the possibility of crosstalk between Nrf2 and cAMP/PKA signaling in adipocytes.

$\beta 3-A R$ stimulation induces CAMP mediated PKA activity and subsequent Creb phosphorylation, leading to transcriptional regulation of target genes containing cAMP-responsive element (CRE) $(10,14,15)$. $\beta 3$-AR can also relay the signaling through other cellular pathways including p38, PKC, and ERK1/2 in adipocytes (27-30). Interestingly, adipocytes exposed to cool temperatures induce Ucp1 expression independent of the PKA/Creb pathway (31), indicating the existence of alternative or parallel pathways for $\beta$-AR mediated Ucp1 induction in adipocytes. Our data show that $\beta 3-A R$ stimulation induces $\mathrm{Nrf} 2$ activation and the Nrf2 can contribute to the increases of Ucp1 expression in adipocytes. Since the adrenergic signaling is often associated with increased production of ROS (32), it is also reasonable that the increased ROS levels by $\beta 3$-AR activation can induce Nrf2 mediated gene expression including Ucp1 and $\mathrm{Nr} 2$ target genes in adipocytes. Therefore, the dissection on the $\beta 3-\mathrm{AR} / \mathrm{CAMP} / \mathrm{PKA} / \mathrm{CREB}$ and the $\beta 3-\mathrm{AR} / \mathrm{Nrf} 2$ signaling cascades for functional relationships should be investigated in future.

We found that $\beta 3$-AR stimulation increased the expression of conventional Nrf2 downstream target genes in an Nrf2-dependent manner. We also presented evidence that the degree of Ucp1 induction by $\beta 3$-AR agonists was partly blunted in the absence of Nrf2 expression in adipocytes. Additionally, the CL316,243induced oxygen consumption was partly impaired in $\mathrm{Nrf} 2 \mathrm{KO}$ obese mice, indicating a role of Nrf2 in $\beta 3$-AR-induced Ucp1 expression in adipocytes and energy expenditure in obese mice. Consistent with our findings, it has been reported that C3H10T1/2 undifferentiated mesenchymal stem cells and bone marrow cells expressed $\beta_{2}$-AR and its activation by adrenaline protected cells from oxidative stress through the Nrf2-mediated induction of glutathione synthesis (33). It is thus possible that adrenergic stimulation-induced Nrf2 activation may not be $\beta$-AR subtype-specific.

At present, it is not clear how the $\beta 3-A R$ signaling pathway regulates Nrf2 activity. Under basal conditions, the Nrf2-Keap1 complex present in the cytosol constantly degrades Nrf2. When Keap1 senses cellular oxidative and electrophilic stress, keap1 and Nrf2 complexes are disrupted, leading to the stabilization and activation of Nrf2. Oxidative/electrophilic stress results in kinase mediated phosphorylation on Nrf2 or Nrf2 negative regulators (i.e. Keap1 and Bach1) for increasing Nrf2 activities. Thus, it is tempting to speculate that $\beta 3$-AR signaling-induced kinase including PKA may affect Nrf2 or its negative regulators to stabilize Nrf2. Future studies are necessary to dissect the exact molecular connection between $\mathrm{Nrf} 2$ and $\beta 3$-AR signaling in adipocytes.

In conclusion, we provide data for new insight into the role of Nrf2 in $\beta 3$-AR-mediated effects and further suggest the utility of Nrf2-mediated thermogenic stimulation as a therapeutic approach for diet-induced obesity.

\section{MATERIALS AND METHODS}

\section{Cell culture and expression analysis}

C3H10T1/2 cells and primary adipocytes were maintained and differentiated as previously reported (34). The adipocytes were treated with $5 \mu \mathrm{M}$ CL316,243 (Sigma, St. Louis, MO, USA) or $10 \mathrm{nM}$ isoproterenol (Sigma) and total RNA was isolated for expression analysis. To measure gene expression levels, total RNA was isolated using TRIzol reagent (Invitrogen, Waltham, MA, USA) and reverse-transcribed using ReverTra Ace ${ }^{\mathbb{R}}$ qPCR RT Master Mix (Toyobo, Japan). Real-time PCR was performed using THUNDERBIRD ${ }^{\mathbb{R}}$ SYBR $^{\circledR}$ qPCR Mix (Toyobo). PCR primers were described previously $(34,35)$. Expression was normalized to the level of ribosomal 36B4 protein as previously described.

\section{Animal studies}

All animal studies were carried out in accordance with the guidelines of the Animal Research Committee (SKKUIACUC202008-21-1) of Sungkyunkwan University. Seven weeks old male C57BL6J mice were purchased from Central Lab Animal Inc (Seoul, KoreA). and housed in rooms at ambient temperature. The Nrf2 KO mice were previously described (35). Whole-body energy metabolism was measured using the Columbus Instruments Oxymax Lab Animal Monitoring System. The mice were placed in metabolic cages and acclimated in the metabolic chambers for one day before measuring $\mathrm{O}_{2}$ consumption.

\section{Statistical analysis}

The data are presented as the mean \pm standard error of the mean (s.e.m). Comparisons between the control and experimental groups were analyzed using unpaired Student's t-tests. Statistical analyses were performed using GraphPad Prism (GraphPad Software, Inc., La Jolla, CA, USA).

\section{ACKNOWLEDGEMENTS}

This study was supported by grants (NRF-2020R111A1A01074938 to U.J.Y., NRF-2020R1A2B5B02001592 to K.W.P.) of the Basic Science Research Program through the National Research Foundation of Korea (NRF) funded by the Ministry of Education, Science, and Technology, Republic of Korea. This study was also partly supported by The Health Fellowship Foundation to S.H.C.

\section{CONFLICTS OF INTEREST}

The authors have no conflicting interests.

\section{REFERENCES}

1. Betz MJ and Enerback S (2018) Targeting thermogenesis in brown fat and muscle to treat obesity and metabolic disease. Nat Rev Endocrinol 14, 77-87 
2. Chang, SH, Song, NJ, Choi JH, Yun UJ and Park KW (2019) Mechanisms underlying UCP1 dependent and independent adipocyte thermogenesis. Obes Rev 20, 241251

3. Fedorenko A, Lishko PV and Kirichok Y (2012) Mechanism of fatty-acid-dependent UCP1 uncoupling in brown fat mitochondria. Cell 151, 400-413

4. Divakaruni AS and Brand MD (2011) The regulation and physiology of mitochondrial proton leak. Physiology (Bethesda) 26, 192-205

5. Vitali A, Murano I, Zingaretti MC, Frontini A, Ricquier D and Cinti $S$ (2012) The adipose organ of obesity-prone C57BL/6J mice is composed of mixed white and brown adipocytes. J Lipid Res 53, 619-629

6. Himms-Hagen J, Melnyk A, Zingaretti MC, Ceresi E, Barbatelli G and Cinti S (2000) Multilocular fat cells in WAT of CL-316243-treated rats derive directly from white adipocytes. Am J Physiol Cell Physiol 279, C670-681

7. Stanford KI, Middelbeek RJ and Goodyear LJ (2015) Exercise effects on white adipose tissue: beiging and metabolic adaptations. Diabetes 64, 2361-2368

8. Yoneshiro T, Ogawa T, Okamoto $\mathrm{N}$ et al (2013) Impact of UCP1 and beta3AR gene polymorphisms on age-related changes in brown adipose tissue and adiposity in humans. Int J Obes (Lond) 37, 993-998

9. Morrison SF, Madden CJ and Tupone D (2012) Central control of brown adipose tissue thermogenesis. Front Endocrinol (Lausanne) 3, 1-19

10. Collins S (2011) Beta-adrenoceptor signaling networks in adipocytes for recruiting stored fat and energy expenditure. Front Endocrinol (Lausanne) 2, 102

11. Cypess AM, Weiner LS, Roberts-Toler C et al (2015) Activation of human brown adipose tissue by a beta3adrenergic receptor agonist. Cell Metab 21, 33-38

12. Matthias A, Ohlson KB, Fredriksson JM, Jacobsson A, Nedergaard J and Cannon B (2000) Thermogenic responses in brown fat cells are fully UCP1-dependent. UCP2 or UCP3 do not substitute for UCP1 in adrenergically or fatty scid-induced thermogenesis. J Biol Chem 275, 25073-25081

13. Inokuma K, Ogura-Okamatsu Y, Toda C, Kimura K, Yamashita $\mathrm{H}$ and Saito M (2005) Uncoupling protein 1 is necessary for norepinephrine-induced glucose utilization in brown adipose tissue. Diabetes 54, 1385-1391

14. Guan XM, Amend A and Strader CD (1995) Determination of structural domains for $G$ protein coupling and ligand binding in beta 3-adrenergic receptor. Mol Pharmacol 48, 492-498

15. Mayr B and Montminy M (2001) Transcriptional regulation by the phosphorylation-dependent factor CREB. Nat Rev Mol Cell Biol 2, 599-609

16. Schneider KS and Chan JY (2013) Emerging role of Nrf2 in adipocytes and adipose biology. Adv Nutr 4, 62-66

17. Shaw P and Chattopadhyay A (2020) Nrf2-ARE signaling in cellular protection: Mechanism of action and the regulatory mechanisms. J Cell Physiol 235, 3119-3130

18. Wakabayashi N, Slocum SL, Skoko JJ, Shin S and Kensler TW (2010) When NRF2 talks, who's listening? Antioxid Redox Signal 13, 1649-1663

19. Lee HI, Lee GR, Lee J et al (2020) Dehydrocostus lactone inhibits NFATC1 via regulation of IKK, JNK, and Nrf2, thereby attenuating osteoclastogenesis. BMB Rep 53, 218-222

20. Jaiswal AK (2004) Nrf2 signaling in coordinated activation of antioxidant gene expression. Free Radic Biol Med 36, 1199-1207

21. Potashnik R, Bloch-Damti A, Bashan $\mathrm{N}$ and Rudich $\mathrm{A}$ (2003) IRS1 degradation and increased serine phosphorylation cannot predict the degree of metabolic insulin resistance induced by oxidative stress. Diabetologia 46 , 639-648

22. Elbini Dhouib I, Jallouli M, Annabi A, Gharbi N, Elfazaa S and Lasram MM (2016) A minireview on N-acetylcysteine: an old drug with new approaches. Life Sci 151, 359-363

23. Kulkarni SR, Donepudi AC, Xu J et al (2014) Fasting induces nuclear factor E2-related factor 2 and ATP-binding Cassette transporters via protein kinase A and Sirtuin-1 in mouse and human. Antioxid Redox Signal 20, 15-30

24. Astort F, Repetto EM, Rocha-Viegas L et al (2016) Role of CREB on heme oxygenase- 1 induction in adrenal cells: involvement of the PI3K pathway. J Mol Endocrinol 57, 113-124

25. Kronke G, Bochkov VN, Huber J et al (2003) Oxidized phospholipids induce expression of human heme oxygenase-1 involving activation of cAMP-responsive elementbinding protein. J Biol Chem 278, 51006-51014

26. Mylroie H, Dumont O, Bauer A et al (2015) PKCepsilonCREB-Nrf2 signalling induces HO-1 in the vascular endothelium and enhances resistance to inflammation and apoptosis. Cardiovasc Res 106, 509-519

27. Tchivileva IE, Tan KS, Gambarian M et al (2009) Signaling pathways mediating beta3-adrenergic receptor-induced production of interleukin-6 in adipocytes. Mol Immunol 46, 2256-2266

28. Lindquist JM, Fredriksson JM, Rehnmark S, Cannon B and Nedergaard J (2000) Beta 3- and alpha1-adrenergic Erk1/2 activation is Src- but not Gi-mediated in Brown adipocytes. J Biol Chem 275, 22670-22677

29. Soeder KJ, Snedden SK, Cao W et al (1999) The beta3adrenergic receptor activates mitogen-activated protein kinase in adipocytes through a $\mathrm{Gi}$-dependent mechanism. J Biol Chem 274, 12017-12022

30. Gerhardt CC, Gros J, Strosberg AD and Issad T (1999) Stimulation of the extracellular signal-regulated kinase $1 / 2$ pathway by human beta-3 adrenergic receptor: new pharmacological profile and mechanism of activation. Mol Pharmacol 55, 255-262

31. Ye L, Wu J, Cohen P et al (2013) Fat cells directly sense temperature to activate thermogenesis. Proc Natl Acad Sci U S A 110, 12480-12485

32. Corbi G, Conti V, Russomanno G et al (2013) Adrenergic signaling and oxidative stress: a role for sirtuins? Front Physiol 4, 324

33. Takahata $Y$, Takarada $T$, lemata $M$ et al (2009) Functional expression of beta2 adrenergic receptors responsible for protection against oxidative stress through promotion of glutathione synthesis after Nrf2 upregulation in undifferentiated mesenchymal C3H10T1/2 stem cells. J Cell Physiol 218, 268-275

34. Song NJ, Choi S, Rajbhandari P et al (2016) Prdm4 induction by the small molecule butein promotes white 
adipose tissue browning. Nat Chem Biol 12, 479-481

35. Bahn G, Park JS, Yun UJ et al (2019) NRF2/ARE pathway negatively regulates BACE1 expression and ameliorates cognitive deficits in mouse Alzheimer's models. Proc Natl Acad Sci U S A 116, 12516-12523 